\title{
Modified-Release Subantimicrobial Dose Doxycycline Enhances Scaling and Root Planing in Subjects With Periodontal Disease
}

Philip M. Preshaw, ${ }^{*}$ M. John Novak, ${ }^{\dagger}$ James Mellonig, ${ }^{\ddagger}$ Ingvar Magnusson, $₫$ Alan Polson,॥

William V. Giannobile, $₫$ Randal W. Rowland, \# John Thomas, ** Clay Walker,§ Dolphus R. Dawson, ${ }^{\dagger}$ Dennis Sharkey, and Mark H. Bradshaw ${ }^{\dagger \dagger}$

Background: Previous studies showed that adjunctive subantimicrobial dose doxycycline (SDD; 20 mg, twice daily) provides significant clinical benefits to scaling and root planing (SRP). A modified-release SDD formulation containing $40 \mathrm{mg}$ doxycycline (SDD-40) to be taken once daily has been developed. The aim of this study was to investigate the efficacy of SDD- 40 when used as an adjunct to SRP for the treatment of periodontitis.

Methods: A 9-month, double-masked, randomized, placebo-controlled, multicenter study was conducted to test the efficacy of adjunctive SDD-40 in 266 subjects with periodontitis. Subjects were treated by SRP and randomized to receive SDD-40 or placebo for 9 months with evaluations at 3, 6 , and 9 months.

Results: Adjunctive SDD-40 provided significantly greater clinical benefits than placebo at all time points. At month 9 , at sites with baseline probing depths (PD) $\geq 6 \mathrm{~mm}, 72 \%$ to $76 \%$ of sites in the SDD-40 group demonstrated clinically significant PD reductions and clinical attachment level (CAL) gains $\geq 2 \mathrm{~mm}$ compared to $56 \%$ to $58 \%$ of sites in the placebo group $(P<0.0001) ; 48 \%$ to $52 \%$ of sites in the SDD-40 group demonstrated PD reductions and CAL gains $\geq 3 \mathrm{~mm}$ compared to $32 \%$ of sites in the placebo group $(P<0.0001)$. In moderate sites (baseline PD 4 to $6 \mathrm{~mm})$, adjunctive SDD-40 provided significant clinical benefits compared to placebo for mean CAL (all time points: $P<0.05$ ), PD (3 months: $P=0.002 ; 6$ and 9 months: $P=0.001$ ), and bleeding on probing (BOP) (3 months: $P<0.01 ; 6$ months: $P<0.02$; 9 months: $P<0.05)$. In deep sites (baseline PD $\geq 7 \mathrm{~mm}$ ), SDD-40 provided significant benefits over control for mean CAL ( 3 months: $P<0.05$; 6 and 9 months: $P<0.01$ ), PD (all time points: $P<0.001$ ), and BOP ( 3 months: $P<0.05$; 6 months: not statistically significant; 9 months: $P<0.05)$. Compliance with study medication was high $(>92 \%)$ with no significant differences in adverse events between groups and no evidence of microbiologically significant changes or development of antibiotic resistance in the subgingival flora in either group.

Conclusion: SDD-40 used as an adjunct to SRP resulted in significantly greater clinical benefits than SRP alone in the treatment of periodontitis. J Periodontol 2008;79:440-452.

\section{KEY WORDS}

Double-masked method; doxycycline/therapeutic use; multicenter; periodontitis/drug therapy.

* Department of Periodontology, School of Dental Sciences, Newcastle University, Newcastle upon Tyne, U.K.

† Department of Periodontology, Center for Oral Health Research, University of Kentucky, Lexington, KY.

$\neq$ Department of Periodontology, University of Texas Health Science Center at San Antonio, San Antonio, TX.

$\S$ Department of Periodontology, University of Florida, Gainesville, FL.

Department of Periodontology, University of Pennsylvania School of Dental Medicine, Philadelphia, PA.

I Department of Periodontology, Michigan Center for Oral Health Research, University of Michigan, Ann Arbor, MI.

\# Private practice, San Francisco, CA.

* * Department of Pathology, West Virginia University, Morgantown, WV.

$\dagger \dagger$ Global Consulting Partners - Medical Biometrics, Princeton, NJ. 
S

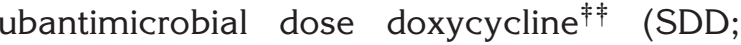
doxycycline, $20 \mathrm{mg}$, twice daily) has become widely established as an effective adjunctive systemic therapy in the management of periodontitis. Clinical studies ${ }^{1-5}$ confirmed that when used as an adjunct to scaling and root planing (SRP) for the treatment of periodontal disease, SDD results in statistically and clinically significant improvements over those achieved by SRP alone. In addition, the long-term use of SDD has been shown to be safe, and it is not associated with the development of antibiotic resistance in the periodontal microflora, ${ }^{6}$ the skin microflora (when used long-term for the management of inflammatory conditions, such as acne), ${ }^{7}$ or the flora of the gastrointestinal or genitourinary tracts. ${ }^{8}$

The clinical benefits of SDD derive from the ability of doxycycline to inhibit the activity of matrix metalloproteinases (MMPs). ${ }^{9}$ MMPs play a key role in periodontal pathogenesis and are secreted by a variety of resident and infiltrating inflammatory cells in diseased periodontal tissues. MMP levels are increased pathologically in inflamed periodontal tissues and in gingival crevicular fluid (GCF) from deep periodontal pockets. ${ }^{10}$ Administration of SDD was shown clearly to result in statistically significant decreases in GCFderived MMPs and bone breakdown components; it also promoted concomitant improvements in the clinical measurements of periodontal status. ${ }^{4,11,12}$ SDD was shown to improve clinical outcomes when used as an adjunct to periodontal flap surgery and to reduce GCF concentrations of markers of alveolar bone resorption. ${ }^{13}$ SDD, by virtue of its anti-inflammatory and anti-MMP effects, was also shown to be of potential benefit in the management of systemic conditions such as coronary artery disease, ${ }^{14}$ rheumatoid arthritis, ${ }^{15}$ and adult acne and rosacea. ${ }^{16}$

The decision whether to use a systemic adjunct to conventional periodontal therapies must be made after consideration of many factors including the diagnosis, the desired treatment outcomes, and the patient's wishes following full explanation of all treatment options. Clearly, the use of a systemic medication over 3 to 9 months requires a commitment on the part of the patient, and poor compliance with a twicedaily medication may affect clinical outcomes. In disorders such as hypertension, it has been shown that compliance with medication is increased as the frequency of daily dosing is decreased. ${ }^{17,18}$ Thus, in terms of compliance, once-daily dosing is preferred to twice-daily (or more) dosing whenever possible.

Although reducing the frequency of dosing enhances compliance and usually is preferred by patients, ${ }^{19}$ this can create challenges in maintaining circulating drug concentrations at the correct therapeutic level. These difficulties may be countered by formulating modified-release systems in which the re- lease and subsequent absorption of a medication is prolonged, thereby ensuring the correct therapeutic dose for an extended period of time. A modifiedrelease formulation of SDD has been developed containing doxycycline monohydrate $40 \mathrm{mg}$ in controlled-release capsules $\S \S$ to be taken once daily (SDD-40). Therefore, the objective of this study was to evaluate the efficacy of SDD-40 when used as an adjunct to SRP compared to placebo for the treatment of periodontitis. Furthermore, the effect, if any, of a 9-month regimen of SDD-40 on the subgingival flora was evaluated compared to the placebo control.

\section{MATERIALS AND METHODS}

\section{Study Design}

This was a randomized, multicenter, double-masked, placebo-controlled parallel group study to evaluate the efficacy of SDD-40 when used as an adjunct to SRP in the treatment of periodontitis. The duration of the study was $\sim 9.5$ months, consisting of a 2 -week screening process and a 9-month treatment period. Subjects were assigned randomly to treatment with SDD-40 or placebo once daily for 9 months. The study was conducted between May 2004 and August 2005 at seven clinical investigational sites (Universities of Kentucky, Texas at San Antonio, Florida, Pennsylvania, Michigan, and Newcastle upon Tyne and a private practice in San Francisco), with all microbiologic assays conducted at West Virginia University. Approval was granted by the respective institutional review boards or ethics committee of the study centers prior to study initiation. Written informed consent was obtained from all subjects. Subjects were screened to confirm eligibility and then underwent full-mouth SRP at the baseline visit. Following this, subjects were allocated randomly to receive adjunctive SDD-40 or adjunctive placebo for the treatment period of the study (9 months) commencing at baseline. Subjects returned to their respective study center for evaluations at 3, 6, and 9 months after baseline.

\section{Study Population}

Eligible subjects were male or female, $\geq 18$ years old, with evidence of untreated periodontitis manifested by four periodontal sites in each of two quadrants, i.e., eight qualifying periodontal sites, with a minimum of two affected teeth per quadrant, with all eight qualifying sites demonstrating probing depth $(P D) \geq 5 \mathrm{~mm}$, attachment loss $\geq 5 \mathrm{~mm}$, and bleeding on probing (BOP) score $^{20} \geq 1$, with at least two sites having bleeding scores $\geq 2$. Exclusion criteria included pregnant or nursing females; serious medical conditions, e.g., kidney or liver disease; systemic infection; dental prophylaxis within the last 30 days; requirement for

キ Periostat, CollaGenex Pharmaceuticals, Newtown, PA. $\S \S$ Oracea, CollaGenex Pharmaceuticals. 
antibiotic prophylaxis prior to dental procedures; use of non-tetracycline antibiotics within 6 weeks of baseline; use of tetracycline antibiotics within 3 months of baseline; hypersensitivity to tetracyclines; a requirement for chronic ( $\geq 2$ weeks) antibiotic therapy; surgery to bypass or exclude the duodenum; and achlorhydria.

\section{Procedures}

Written informed consent was obtained at the screening examination. A health history was recorded, and an oral pathology examination was undertaken. Fullmouth clinical attachment level (CAL) and PD measures were recorded by trained examiners using manual University of North Carolina 15 periodontal probes at six sites per tooth. BOP was recorded at six sites per tooth using a bleeding index $(0=$ no bleeding; 1 = single bleeding point or fine line of blood; 2 = interdental triangle or direct margin fills with blood; and 3 = profuse bleeding immediately after probing). ${ }^{20}$ Venous blood samples were obtained, and a pregnancy test was performed when appropriate to exclude pregnancy. Radiographs taken within 12 months of screening (including any taken at the screening appointment itself) were used to confirm the periodontal diagnosis and identify any underlying conditions that might exclude a subject from participation in the study. Demographic information, details of oral hygiene practices, and smoking histories were recorded.

The screening examination also served as the baseline examination for those subjects who immediately qualified for the study. Otherwise, subjects returned for the baseline examination 14 days after screening. In either case, following the baseline examination, full-mouth SRP was undertaken commencing immediately after the baseline examination and completed within a maximum of 24 hours. SRP was performed by supra- and subgingival use of ultrasonic instruments followed by hand instruments for removal of plaque and calculus deposits until tooth surfaces were smooth and calculus could not be detected, with a time limit of 1 hour per quadrant. Ultrasonic and universal or area-specific curets were used, as was local anesthesia, based on operator preference.

At baseline, subjects were randomized to receive SDD-40 or placebo once daily (1:1 randomization). Randomization was carried out at each investigational site based on a randomly generated predefined schedule with fixed block size. Subjects were instructed to take study medication with water $\geq 1$ hour before or 2 hours after meals. Subjects were instructed that antacids and nutritional supplements containing $\mathrm{Al}, \mathrm{Ca}$, or Mg may impair drug absorption and that these were to be taken $\geq 1.5$ hours before or 3 hours after taking study medication. Study medication and placebo were identical in appearance and were dispensed in 3-month quantities. All personnel involved in the study were masked to study medication allocation. Subjects returned for full-mouth clinical assessments at 3, 6, and 9 months and were contacted monthly by telephone for the recording of adverse events (AEs) and for ensuring compliance. At month 9, a dental prophylaxis was performed, and the subjects were exited from the study.

\section{Assessments}

Prior to the study, all examiners were trained and practiced in each of the clinical measurements. To minimize the impact of interexaminer variability, the same examiner assessed a given subject for the duration of the study. Prior to the primary statistical analyses, sites were stratified by the degree of PD at baseline: sites with PD of 1 to $3 \mathrm{~mm}$ were considered normal; sites with PD of 4 to $6 \mathrm{~mm}$ were considered mild-moderately diseased; and sites with baseline PD $\geq 7 \mathrm{~mm}$ were considered severely diseased. ${ }^{21}$

Full-mouth PD, BOP, and CAL were recorded at baseline and months 3, 6, and 9. Efficacy parameters included the mean changes in PD, BOP, and CAL score from baseline. AEs and the use of concomitant medications were documented in subject diaries and recorded at each study visit and during monthly telephone contacts. Compliance with study drug therapy was recorded by counting the number of tablets dispensed and returned. Laboratory tests (blood chemistry and complete blood cell count and differentiation) were conducted at the screening/baseline visit and month 9. Vital signs, oral soft tissue examinations, and examinations of the head and neck were performed at screening/baseline and at months 3, 6, and 9 . The pregnancy test was repeated at month 9 when appropriate.

\section{Microbiologic Assessments}

At two preselected investigational centers (University of Kentucky and University of Florida), subgingival plaque samples were collected using sterile paper points at baseline and month 9 and analyzed for microflora and antibiotic susceptibility. Plaque was collected from four of the eight selected qualifying sites with PD $\geq 5 \mathrm{~mm}$ and $\leq 8 \mathrm{~mm}$. Obvious supragingival plaque was removed and discarded. Subgingival samples were collected by inserting a single sterile endodontic paper point into the pocket until resistance was felt. The paper point was moved laterally parallel to the long axis of the tooth and left in place for $\sim 10$ seconds as described previously. ${ }^{6}$ The point was removed and immediately placed in modified Amies transport medium. ${ }^{22}$ A separate paper point was used to sample each site, but the sites were pooled by subject for processing.

For analysis (undertaken at West Virginia University), samples were dispersed by low wattage 
sonication and vortexing for 10 seconds using anaerobic techniques. Ten-fold serial dilutions were made under anaerobic conditions using prereduced anaerobically sterilized (PRAS) Ringers solution, and 0.1$\mathrm{ml}$ aliquots were dispensed onto the surface of agar plates and spread with sterile glass rods. Following the prescribed incubation period, plates were examined for colony forming units (CFU). Counts for the enumeration of total anaerobic and total facultative counts were performed on the plate dilutions that gave rise to 20 to $200 \mathrm{CFU}$. Counts on selective media were performed using the plate dilutions that yielded 20 to 200 CFU.

To determine the effect of SDD-40 on the total cultivable flora, $0.1-\mathrm{ml}$ aliquots of the diluted samples were spread onto trypticase-soy blood agar supplemented with hemin and menadione (TSBA-HK) medium containing $4 \mu \mathrm{g}$ doxycycline per milliliter of medium (TSBA + doxycycline). These plates were incubated anaerobically along with the TSBA-HK plates for the determination of total anaerobic bacterial counts. Following 5 to 7 days of incubation, both sets of plates (TSBA-HK doxycycline containing and nondoxycycline containing) were examined for CFU. The colonies on the countable TSBA + doxycycline plates were examined; colonies belonging to each of the most numerous colony types present (up to three different colony types per sample when present) were counted, and a representative isolate of each was subcultured to TSBA-HK. Each isolate that survived subculture was identified to genus and species level. The minimal inhibitory concentrations (MICs) of doxycycline, minocycline, tetracycline, erythromycin, clindamycin, and amoxicillin were determined by agar dilution or by the E-test for each isolate that survived the characterization and identification process. ${ }^{6,23}$

This procedure yielded the following: percentage of the cultivable flora resistant to doxycycline, the identity of the predominant doxycycline-resistant flora, and the presence or absence of multiantibiotic resistance and the particular antibiotics to which the microorganism was resistant. The percentage of cultivable flora resistant to doxycycline was calculated from the total counts on doxycycline-containing medium as a percentage of the total anaerobic counts on non-doxycycline-containing medium.

\section{Statistical Analyses}

The study was designed to detect a difference $\geq 0.2$ $\mathrm{mm}$ in mean change from baseline CAL between treatment arms (two-tailed tests) with $80 \%$ power, assuming that 72 subjects would complete the study in each group. An intent-to-treat analysis strategy was used for all subjects who were randomized to study medication and who took at least one dose. For subjects not completing the study, a last-observation- carried-forward (LOCF) algorithm was used to impute missing data at each time point.

Changes in mean CAL, PD, and BOP were calculated at each time point from subject means categorized according to PD at baseline. Comparisons between the treatment groups were made using analysis of covariance (ANCOVA). The ANCOVA model analyzed the subject-level mean changes from baseline; included factors for treatment group, investigational site, and the treatment by investigational site interaction; and included the baseline value of the parameter for each subject as covariate. All tests of significance were two-sided; differences were considered statistically significant when $P<0.05$.

For sites requiring additional periodontal therapy during the course of the study (those sites that demonstrated $\geq 2 \mathrm{~mm}$ attachment loss at any point during the study), the post-baseline observations recorded immediately prior to the local therapy were analyzed using the LOCF strategy to ensure analyses were conservative and not biased. Differences between the groups for threshold changes indicating disease progression, i.e., $\geq 2 \mathrm{~mm}$ attachment loss, were analyzed using a generalized estimating equations (GEE) analysis for the repeated measures across visits.

To assess the clinical significance of any improvements in periodontal status as a result of treatment, the number of sites achieving clinically significant reductions in PD, i.e., reductions $\geq 2$ and $\geq 3 \mathrm{~mm}$, and clinically significant gains in CAL, i.e., gains $\geq 2$ and $\geq 3 \mathrm{~mm}$, were determined. Separate analyses were done for all sites with baseline PD $\geq 4 \mathrm{~mm}$ and for deeper sites with baseline PD $\geq 6 \mathrm{~mm}$. The proportion of sites achieving such improvements in each group was expressed as a percentage of the total number of sites, and comparisons between the two treatment groups were made using a GEE model with adjustment for within-subject correlations among sites.

Compliance with study medication was defined as the number of medication doses taken as a percentage of the number that should have been taken based on the number of days between study visits. Noncompliance was defined if compliance was $\leq 80 \%$.

The microbial data from both clinical centers that collected plaque samples were combined and analyzed as a single data set. Inferential analyses of doxycycline resistance were based on the per-subject mean percentage of colonies at each visit that showed resistance to doxycycline and the change from baseline in the mean percentage of resistant organisms. Inferential analyses of species-specific colony counts were based on log (10)-transformed counts, where the log (10) transformation of a count of zero was set to zero. The primary analyses sought to determine whether there were statistically significant differences between active and placebo groups in changes from 
baseline to month 9 with regard to the mean percentage of doxycycline-resistant organisms. Separate analyses were conducted for each of the visits and for the changes from baseline to month 9 using the Wilcoxon two-sample test. This non-parametric test was chosen because distributions of the per-subject percentage of resistance data did not meet the requirements for parametric analyses. It is a conservative test that provides unbiased probability estimates under these circumstances. The secondary analyses were performed to determine whether colony counts for specific species were different between the two treatment groups at either visit and whether the changes from baseline in mean log (10) counts differed between treatment groups. As in the case of the primary analyses, separate analyses were conducted for each of the visits and for the changes from baseline using the Wilcoxon two-sample test. In all cases, two-tailed $P$ values $\leq 0.05$ were considered statistically significant. All analyses were performed using a software program.|l||

\section{RESULTS}

A total of 266 subjects were enrolled into the study; 133 were randomized to receive adjunctive SDD-40, and 133 were randomized to receive adjunctive placebo. A total of 110 subjects (82.7\%) in the SDD-40 group and 117 subjects (88.0\%) in the placebo group completed the study. The primary reasons for discontinuing participation were loss to follow-up (SDD-40 group: 10 subjects; placebo group: four subjects), AEs (SDD-40 group: seven subjects; placebo group: four subjects) or lack of compliance (SDD-40 group: two subjects; placebo group: four subjects). There were no statistically significant differences between the SDD-40 and placebo groups with respect to the numbers of subjects who did not continue the study or the reasons for discontinuation.

There were no statistically significant differences between the treatment groups regarding demographic characteristics (Table 1). Similarly, there were no significant differences between the study groups with respect to oral hygiene practices. On average, subjects brushed their teeth twice per day and flossed just over three times per week. Eighteen of 266 (6.8\%) subjects had diabetes; one subject had type 1 diabetes mellitus (this subject was randomized to receive placebo), and 17 had type 2 diabetes mellitus (nine subjects in the SDD-40 group and eight subjects in the placebo group). There were no statistically significant differences in diabetes status between the treatment groups $(P=0.59)$. The treatment groups also were similar with regard to other baseline variables, including medical history, medication history, and use of concomitant medications. Compliance with the study medications was high, with $92 \%$ com-
Table I.

\section{Demographic Characteristics of Study Population}

\begin{tabular}{|c|c|c|c|}
\hline Characteristic & $\begin{array}{c}\text { SRP + SDD-40 } \\
\quad(n=133)\end{array}$ & $\begin{array}{c}\text { SRP + Placebo } \\
\quad(n=133)\end{array}$ & $P$ \\
\hline \multicolumn{4}{|l|}{ Age (years) } \\
\hline Mean \pm SD & $48.5 \pm 11.4$ & $49.9 \pm 11.0$ & 0.34 \\
\hline Range & 24 to 81 & 23 to 82 & \\
\hline \multicolumn{4}{|l|}{ Gender (n [\%]) } \\
\hline Male & $58(43.6)$ & $62(46.6)$ & 0.62 \\
\hline Female & $75(56.4)$ & $71(53.4)$ & \\
\hline \multicolumn{4}{|l|}{ Race/ethnicity (n [\%]) } \\
\hline White & $87(65.4)$ & $100(75.2)$ & 0.90 \\
\hline Black & $28(21.1)$ & $21(15.8)$ & \\
\hline Asian & $8(6.0)$ & $7(5.3)$ & \\
\hline Other & $10(7.5)$ & $5(3.7)$ & \\
\hline \multicolumn{4}{|l|}{ Tobacco use (n [\%]) } \\
\hline Ex- or current smoker & $82(62.6)$ & $84(62.2)$ & 0.95 \\
\hline Current smoker & $45(34.4)$ & $32(23.7)$ & 0.06 \\
\hline
\end{tabular}

$P$ values were determined using the $\chi^{2}$ test for discrete variables and the two-sample $t$ test for continuous variables.

Table 2.

$$
\begin{aligned}
& \text { Baseline Clinical Characteristics } \\
& \text { (mean } \pm \text { SEM) of Study Population }
\end{aligned}
$$

\begin{tabular}{llccc}
\hline Baseline PD & Parameter & SRP + SDD-40 & SRP + Placebo & $P$ \\
\hline 4 to $6 \mathrm{~mm}$ & PD (mm) & $4.91 \pm 0.02$ & $4.89 \pm 0.02$ & 0.46 \\
& CAL (mm) & $4.81 \pm 0.09$ & $4.82 \pm 0.09$ & 0.78 \\
& BOP & $1.89 \pm 0.04$ & $1.88 \pm 0.04$ & 0.81 \\
$\geq 7 \mathrm{~mm}$ & PD (mm) & $7.43 \pm 0.06$ & $7.47 \pm 0.06$ & 0.62 \\
& CAL (mm) & $7.18 \pm 0.16$ & $7.30 \pm 0.15$ & 0.60 \\
& BOP & $2.14 \pm 0.04$ & $2.10 \pm 0.05$ & 0.51 \\
\hline
\end{tabular}

pliance in the SDD-40 group and 95\% compliance in the placebo group.

Baseline clinical parameters are shown in Table 2. There were no statistically significant differences in the baseline clinical characteristics between the two treatment groups.

\section{Clinical Outcomes}

The mean per-subject changes in CAL from baseline to month 9 are shown in Figures 1 and 2. Attachment gains were demonstrated in both groups, which was expected following SRP. However, gains in CAL were significantly greater at all time points in the subjects treated with SDD-40 compared to the subjects who received placebo $(P<0.05)$ whether considering moderate

||| SAS version 8.2, SAS Institute, Cary, NC. 


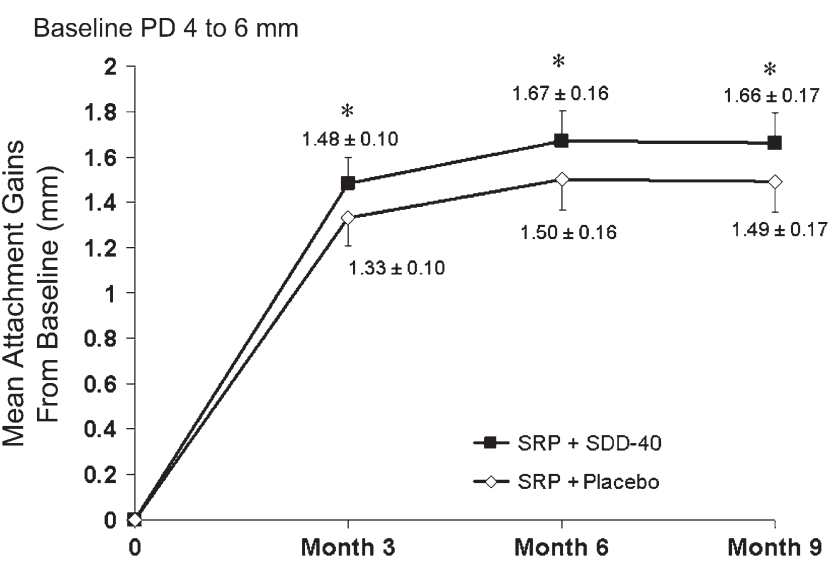

Figure I.

Mean attachment gains in sites with baseline PD of 4 to $6 \mathrm{~mm}$. The mean per-subject changes from baseline and standard errors are presented. *P $<0.05$ versus placebo.

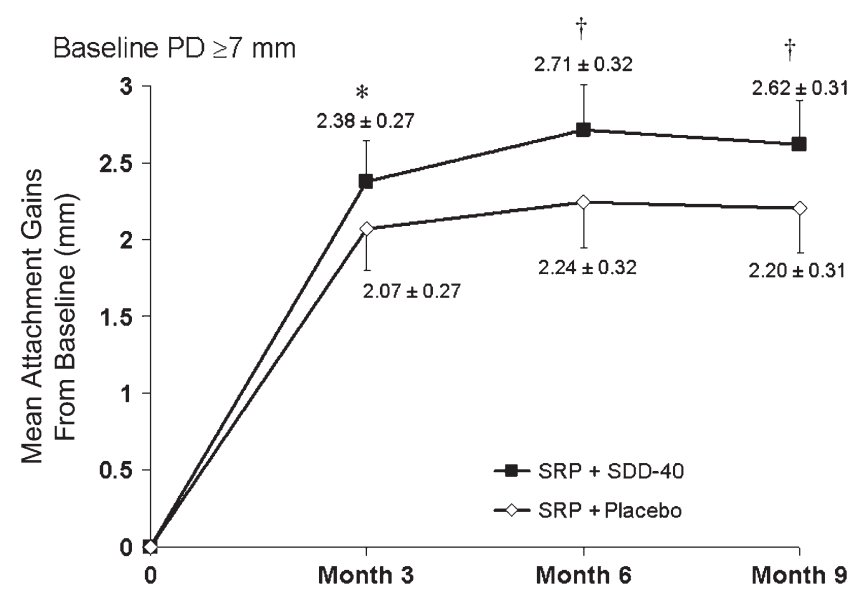

Figure 2.

Mean attachment gains in sites with baseline PD $\geq 7 \mathrm{~mm}$. The mean per-subject changes from baseline and standard errors are presented. *P $<0.05$ versus placebo; ${ }^{\dagger} \mathrm{P}<0.0$ I versus placebo.

(baseline PD 4 to $6 \mathrm{~mm}$ ) or deep (baseline PD $\geq 7 \mathrm{~mm}$ ) sites. In moderate sites at month 9 , mean CAL gains from baseline were $11 \%$ greater with adjunctive SDD40 than with placebo $(1.66 \mathrm{~mm}$ versus $1.49 \mathrm{~mm}$, respectively; $P=0.02$ ). In deep sites at month 9 , mean CAL gains from baseline were $19 \%$ greater with adjunctive SDD-40 than with placebo $(2.62 \mathrm{~mm}$ versus $2.20 \mathrm{~mm}$, respectively; $P<0.01)$.

The mean per-subject changes in PD from baseline to month 9 are shown in Figures 3 and 4 . Again, as was expected following SRP, PD reductions were observed in both groups. These were significantly greater at all time points, at moderate and deep sites, in the subjects treated with SDD-40 compared to the subjects who received placebo $(P<0.01)$. In moderate sites

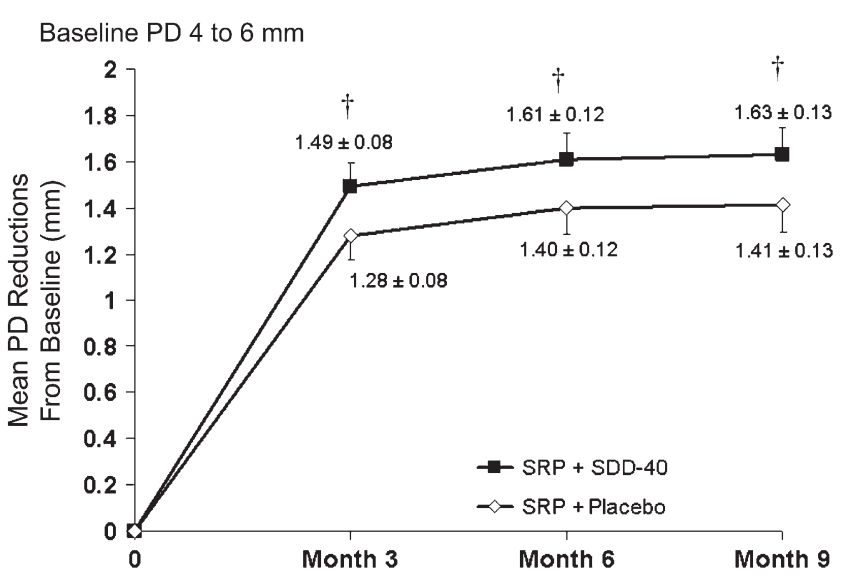

Figure 3.

Mean PD reductions in sites with baseline PD of 4 to $6 \mathrm{~mm}$. The mean per-subject changes from baseline and standard errors are presented. $t_{\mathrm{P}}<0.01$ versus placebo.

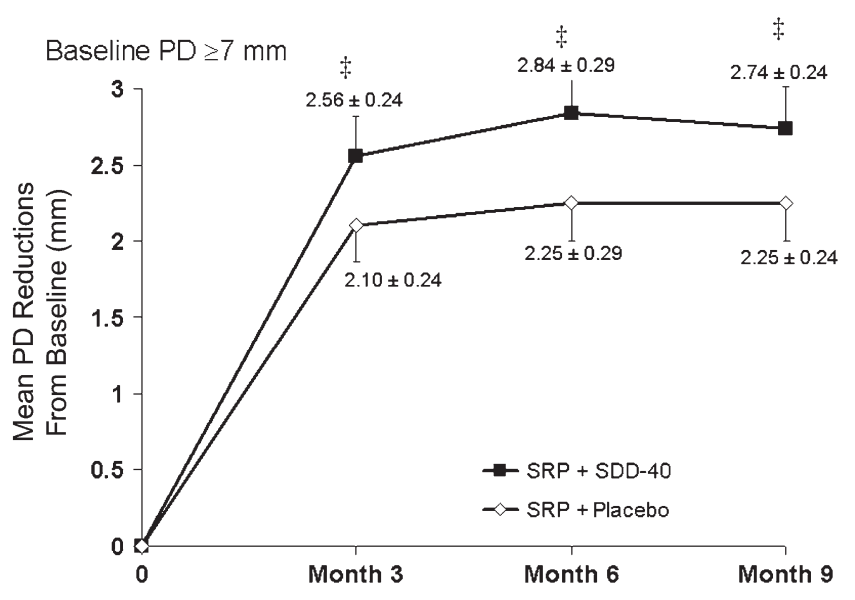

Figure 4.

Mean PD reductions in sites with baseline $P D \geq 7 \mathrm{~mm}$. The mean per-subject changes from baseline and standard errors are presented. ${ }^{\ddagger} \mathrm{P}<0.00$ I versus placebo.

at month 9, mean PD reductions from baseline were $16 \%$ greater with adjunctive SDD-40 than with placebo (1.63 mm versus $1.41 \mathrm{~mm}$, respectively; $P<0.01)$. In deep sites at month 9, mean PD reductions from baseline were $22 \%$ greater with adjunctive SDD-40 than with placebo (2.74 mm versus $2.25 \mathrm{~mm}$, respectively; $P<0.001)$.

Although mean changes in PD and CAL are useful summary statistics, they do not lend themselves easily to interpretation of whether improvements are clinically significant. Therefore, a threshold analysis was undertaken to determine the proportion of sites undergoing PD reductions and CAL gains $\geq 2$ and $\geq 3 \mathrm{~mm}$ in the two treatment groups. Table 3 presents the results of this analysis and confirms that, in every 
Table 3.

\section{Threshold Analyses of Sites Demonstrating Clinically Significant CAL Gains and PD Reductions at Month 9}

\begin{tabular}{lcccc}
\hline & \multicolumn{2}{c}{ All Sites (baseline PD $\geq 4 \mathrm{~mm})$} & \multicolumn{2}{c}{ Deep Sites (baseline PD $\geq 6 \mathrm{~mm})$} \\
\cline { 2 - 5 } $\begin{array}{l}\text { Threshold Change } \\
\text { From Baseline }\end{array}$ & $\begin{array}{l}\text { SRP }+ \text { SDD-40 } \\
(\mathrm{n}=8,260)(\%[\mathrm{n}])\end{array}$ & $\begin{array}{c}\text { SRP + Placebo } \\
(\mathrm{n}=8,046)(\%[\mathrm{n}])\end{array}$ & $\begin{array}{c}\text { SRP + SDD-40 } \\
(\mathrm{n}=2,748)(\%[\mathrm{n}])\end{array}$ & $\begin{array}{c}\text { SRP + Placebo } \\
(\mathrm{n}=2,630)(\%[\mathrm{n}])\end{array}$ \\
\hline CAL gain $\geq 2 \mathrm{~mm}$ & $57.3^{*}(4,732)$ & $45.8(3,688)$ & $71.8^{\dagger}(1,973)$ & $55.9(1,469)$ \\
CAL gain $\geq 3 \mathrm{~mm}$ & $28.3^{*}(2,337)$ & $19.5(1,569)$ & $47.3^{\dagger}(1,299)$ & $31.6(831)$ \\
PD reduction $\geq 2 \mathrm{~mm}$ & $57.7^{*}(4,769)$ & $45.1(3,626)$ & $75.9^{\dagger}(2,086)$ & $57.8(1,520)$ \\
PD reduction $\geq 3 \mathrm{~mm}$ & $25.4^{*}(2,069)$ & $16.5(1,324)$ & $51.7^{\dagger}(1,420)$ & $32.1(845)$ \\
\hline
\end{tabular}

* $P<0.01$ compared to placebo (determined using GEE model with adjustment for within-subject correlations among sites).

$\dagger P<0.0001$ compared to placebo (determined using GEE model with adjustment for within-subject correlations among sites).

instance, a substantial and highly statistically significant benefit of SDD-40 was apparent. For example, when considering the most diseased sites (baseline PD $\geq 6$ $\mathrm{mm}), \sim 48 \%$ to $52 \%$ of sites in the SDD-40 group demonstrated highly clinically significant PD reductions and CAL gains $\geq 3 \mathrm{~mm}$ compared to $\sim 32 \%$ of sites in the placebo group $(P<0.0001)$. Similarly, $\sim 72 \%$ to $76 \%$ of sites with baseline PD $\geq 6$ $\mathrm{mm}$ in the SDD-40 group demonstrated clinically significant PD reductions and CAL gains $\geq 2 \mathrm{~mm}$ compared to $56 \%$ to $58 \%$ of sites in the placebo group $(P<0.0001)$.

Mean changes in BOP scores are presented in Table 4. Reductions in mean BOP scores were observed in both treatment groups following SRP, as was expected. However, reductions in BOP were significantly greater in the SDD-40 group than the placebo group at all time points in moderate and deep sites (with the exception of deep sites at month 6 , at which there was no significant difference between SDD-40 and placebo).

The percentage of sites demonstrating progressing disease manifested by attachment loss $\geq 2$ or $\geq 3 \mathrm{~mm}$ from baseline are presented in Table 5. There was a tendency for subjects in the SDD-40 group to demonstrate a slightly lower percentage of sites with attach-

Table 4.

* $P<0.01$ compared to placebo.

\section{Table 5.}

Changes (mean \pm SEM) in BOP From Baseline

\begin{tabular}{lcccc}
\hline & SRP + SDD-40 & SRP + Placebo & SRP + SDD -40 & SRP + Placebo \\
\cline { 2 - 5 } Month & Moderate Sites (PD 4 to $6 \mathrm{~mm})$ & Deep Sites $(P D \geq 7 \mathrm{~mm})$ \\
\hline 3 & $-1.19 \pm 0.13^{*}$ & $-1.07 \pm 0.13$ & $-1.20 \pm 0.16^{\dagger}$ & $-1.03 \pm 0.16$ \\
6 & $-1.26 \pm 0.13^{\dagger}$ & $-1.16 \pm 0.13$ & $-1.31 \pm 0.13$ & $-1.16 \pm 0.13$ \\
9 & $-1.27 \pm 0.12^{\dagger}$ & $-1.19 \pm 0.12$ & $-1.31 \pm 0.14^{\dagger}$ & $-1.15 \pm 0.14$ \\
\hline
\end{tabular}

$\dagger P<0.05$ compared to placebo.

Negative numbers indicate reduction in BOP

\section{Per-Subject Percentage (mean \pm SEM) of Sites Demonstrating} Attachment Loss $\geq 2$ and $\geq 3 \mathrm{~mm}$ From Baseline

\begin{tabular}{lccccc}
\hline & & SRP + SDD-40 & SRP + Placebo & SRP + SDD-40 & SRP + Placebo \\
\cline { 2 - 5 } Attachment Loss & Month & Moderate Sites (PD 4 to 6 mm) & \multicolumn{1}{c}{ Deep Sites (PD $\geq 7 \mathrm{~mm})$} \\
\hline $22 \mathrm{~mm}$ & 3 & $1.2 \pm 0.5$ & $1.4 \pm 0.5$ & $0.8 \pm 0.5$ & $1.9 \pm 0.5$ \\
& 6 & $1.2 \pm 0.5$ & $1.5 \pm 0.5$ & $0.9 \pm 0.6$ & $1.9 \pm 0.6$ \\
& 9 & $1.4 \pm 0.6$ & $1.7 \pm 0.5$ & $2.1 \pm 0.9$ & $2.1 \pm 0.9$ \\
& 3 & $0.3 \pm 0.2$ & $0.4 \pm 0.2$ & $0.4 \pm 0.3$ & $0.9 \pm 0.3$ \\
& 6 & $0.3 \pm 0.2$ & $0.6 \pm 0.2$ & $0.5 \pm 0.4$ & $1.0 \pm 0.4$ \\
& 9 & $0.6 \pm 0.3$ & $0.9 \pm 0.3$ & $0.3 \pm 0.4$ & $1.2 \pm 0.4$ \\
\hline
\end{tabular}

ment loss $\geq 2$ and $\geq 3 \mathrm{~mm}$ from baseline compared to the placebo group, but these differences were not statistically significant.

\section{Safety Data}

In the SDD-40 group, 88 subjects $(66.2 \%)$ reported a total of 217 AEs; 94 subjects (70.7\%) reported a total of $229 \mathrm{AEs}$ in the placebo group. In the SDD-40 group, the most frequently reported AEs were 
headache ( 13 subjects; $9.8 \%$ ) and influenza and nasopharyngitis (each seven subjects; $5.3 \%$ ). In the placebo group, the most frequently reported AEs were sensitivity of teeth (13 subjects; $9.8 \%$ ) and headache and nasopharyngitis (each 10 subjects; $7.5 \%$ ). AEs were ranked as mild, moderate, or severe in intensity, with the most common being mild or moderate. Severe AEs were recorded for nine (6.8\%) subjects in the SDD-40 group and 18 (13.5\%) subjects in the placebo group. No AE was considered to be probably related to the study medication in either group. Serious AEs (SAEs) were reported for six (4.5\%) subjects in the SDD-40 group and three (2.3\%) subjects in the placebo group, and no SAE was considered to be related to treatment. Adjunctive SDD-40 was tolerated well, and there were no clinically meaningful differences in the numbers of AEs between the treatment groups, including any associated with the gastrointestinal tract or genitourinary tract.

No clinically meaningful differences between the placebo and SDD groups were found for any laboratory tests (complete blood cell count and blood chemistry), and there were no notable mean changes from baseline to month 9 in either treatment group for any laboratory parameter. No positive pregnancy tests were recorded, and there were no differences between the treatment groups in changes in vital signs or weight.

\section{Microbiologic Outcomes}

Data from 70 subjects (34 in the SDD-40 group and 36 in the placebo group) were available for microbiologic analysis. It was immediately apparent that an imbalance existed between the two treatment groups in the percentage resistance to doxycycline at the baseline visit, and subjects randomized to SDD-40 presented with a three-fold greater load of doxycycline-resistant bacteria $(12.69 \%)$ than did subjects randomized to placebo (3.95\%) (Table 6). A similar imbalance also was present at month 9 when twice as many subjects in the SDD-40 group demonstrated resistance to doxycycline compared to the placebo group. However, when considering the change in doxy-

\section{Table 6.}

Percentage (mean \pm SD) of Recovered Flora Resistant to Doxycycline $(4 \mu \mathrm{g} / \mathrm{ml})$ for Each Treatment Group

\begin{tabular}{lrc}
\hline & SDD-40 & Placebo \\
\hline Baseline & $12.69 \pm 23.16$ & $3.95 \pm 7.39$ \\
Month 9 & $17.79 \pm 20.85$ & $9.33 \pm 20.64$ \\
$\begin{array}{l}\text { Change from baseline } \\
\text { to month 9 }\end{array}$ & $5.09 \pm 31.17$ & $5.38 \pm 22.02$ \\
\hline
\end{tabular}

cycline-resistant counts over the course of the study, the two groups were very similar, with an increase of just $>5 \%$ in each treatment group. There were no statistically significant differences by parametric or nonparametric analysis over time $(P=0.965$, analysis of variance; $P=0.156$, Wilcoxon).

The effect of treatment, i.e., SDD-40 versus placebo, on the specific microbial taxa also was enumerated. The microbial counts were log transformed (log base 10) and then analyzed for differences at baseline (imbalances) and at 9 months; because of the presence of an imbalance between treatment groups at baseline, the change from baseline to 9 months also was analyzed. These data are summarized in Table 7. Statistical analysis of the microbial counts in the two treatment groups revealed that there were no significant differences between the treatment groups at baseline, month 9, or for the change over time (Wilcoxon two-sample test). There were isolated statistically significant differences between the groups, including a statistically significant difference between treatment groups at baseline for Tannerella forsythia (previously $T$. forsythensis) $(P=0.028)$ but not at month $9(P=0.647)$. Statistically significant differences were detected for Porphyromonas gingivalis $(P g)$ at month $9(P=0.043)$ and when change over time was examined $(P=0.025)$. These differences were due to the detection of $\mathrm{Pg}$ in a single subject (700 CFU) at baseline in the SDD-40 group and in a single subject $(50,000 \mathrm{CFU})$ in the placebo group at month 9.

The only other incidence of statistical significance occurred with Aggregatibacter actinomycetemcomitans (Aa) (previously Actinobacillus actinomycetemcomitans). No differences were detected at baseline between the two treatment groups $(P=0.356)$, but a trend toward statistical significance was detected at month $9(P=0.077)$, and significance was detected in the change over time analysis $(P=0.002)$. In the SDD-40 group, Aa was recovered in eight subjects at baseline and nine subjects at month 9 . In the placebo group, the respective numbers were 14 subjects and eight subjects. Thus, the significance in change over time was due to a decrease in Aa in the placebo group and not due to an increase in the SDD-40 group.

To evaluate whether treatment with SDD-40 or placebo resulted in development of doxycycline or antibiotic resistance, bacteria that were recovered on medium containing $4 \mu \mathrm{g} / \mathrm{ml}$ doxycycline were subcultured, identified to genus and species by accepted microbiology methods, and then the MICs of the isolates were determined for doxycycline, minocycline, tetracycline, erythromycin, clindamycin, and amoxicillin. MIC data are not readily analyzable by statistical methods because each antibiotic concentration tested represents a two-fold increase. Thus, MIC differences 
Table 7.

\section{Means $(\log 10)$ of Microbial Counts at Baseline and 9 Months by Treatment Group and Change From Baseline to 9 Months}

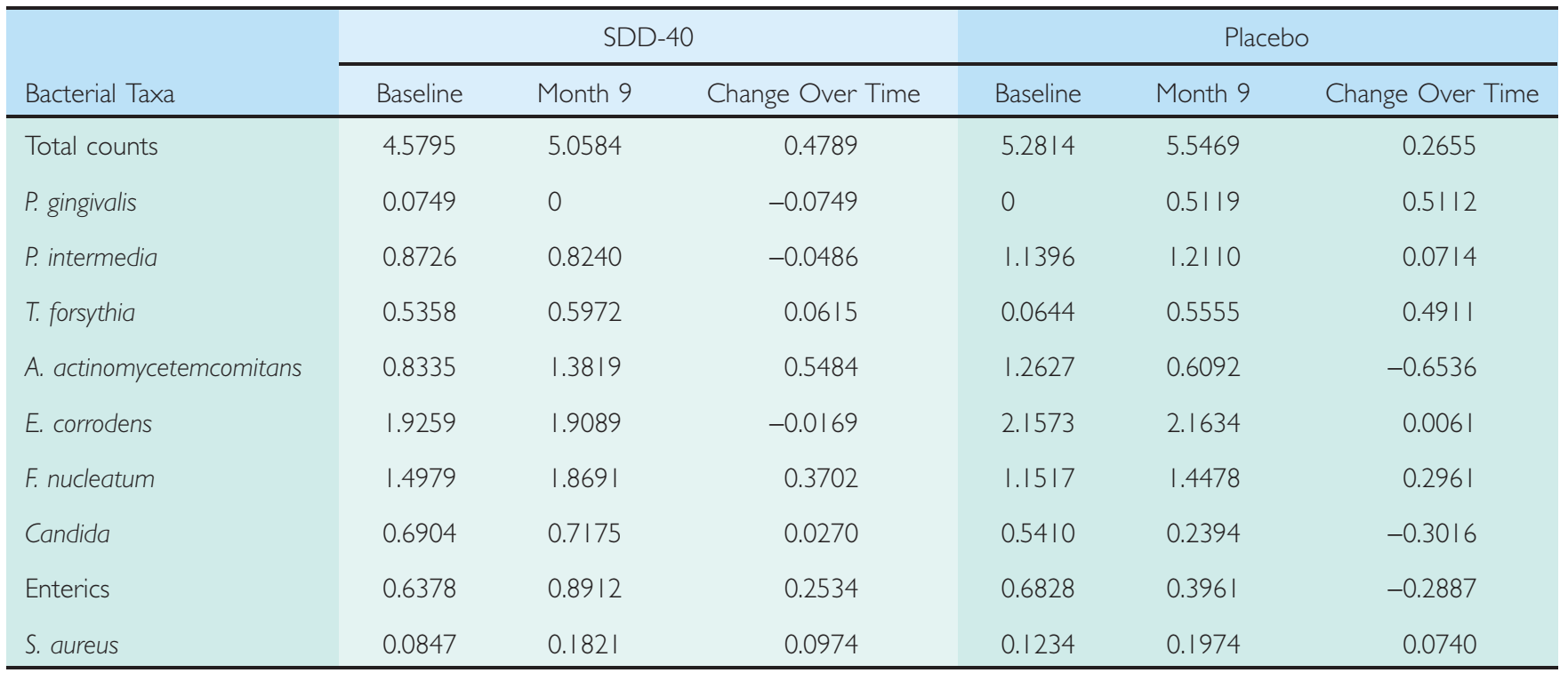

P. intermedia = Prevotella intermedia; E. corrodens = Eikenella corrodens; F. nucleatum = Fusobacterium nucleatum; S. aureus = Staphylococcus aureus. $0=$ none detected; negative value $=$ decrease over time.

\section{Table 8.}

\section{Correlation Coefficients for Correlation of Resistance to Antibiotics With Resistance to Doxycycline for Each Treatment and Sample Period}

\begin{tabular}{lccccc}
\hline & Amoxicillin & Erythromycin & Clindamycin & Minocycline & Tetracycline \\
\hline SDD-40: baseline & 0.4225 & 0.1652 & 0.2294 & 0.3778 & 0.2559 \\
SDD-40: month 9 & 0.4945 & 0.2315 & 0.2660 & 0.1593 & 0.1208 \\
Placebo: baseline & 0.0927 & 0.2761 & 0.4373 & 0.6487 & 0.4322 \\
Placebo: month 9 & 0.3738 & 0.2451 & 0.4480 & 0.6158 & 0.5137 \\
\hline
\end{tabular}

of a single two-fold dilution are not considered microbially significant but would yield statistical significance by parametric or non-parametric testing. Such data are handled best by considering overall changes in the bacterial profiles that show resistance and by examining correlation coefficients to determine whether resistance to one agent promotes resistance to a different agent.

Thus, a correlation matrix (Table 8 ) was created based on the MICs obtained for the doxycycline-resistant bacteria and their susceptibilities to doxycycline and five other antibiotics. Because an imbalance was found in the percentage resistance to doxycycline at baseline for the two treatment groups, attention should be directed at the comparison of correlation coefficients within treatments rather than between treatments. The only instance in which there was a microbiologically significant change was with amoxicillin in the placebo group: a coefficient of 0.0927 was obtained for the baseline data, and a coefficient of 0.3738 was obtained at 9 months. There were no strong coefficients of correlation $(>0.5)$ obtained between doxycycline resistance and resistance to any of the other five antibiotics in the SDD-40 treatment group.

Finally, Figure 5 provides a graphic representation of the major taxonomic groups recovered and the proportion that each contributed to the total doxycyclineresistant profile. Although biologic system variation is seen, there were no microbiologically significant changes in the profiles obtained over time for the SDD-40 group or the placebo group. 


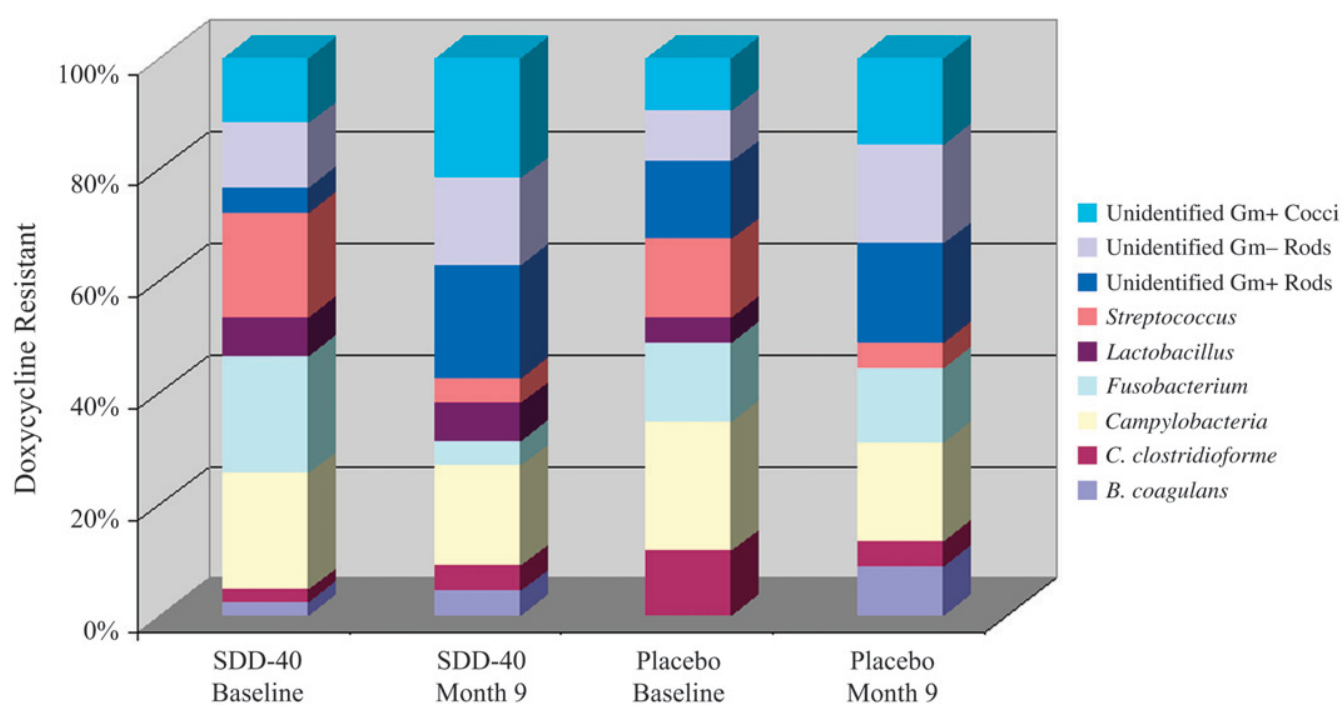

Figure 5.

Profiles of bacterial taxa recovered at each sample period of each treatment that demonstrated resistance to $4 \mu \mathrm{g} / \mathrm{ml}$ doxycycline. Gm+ = Gram positive; Gm- = Gram negative; C. clostridioforme = Clostridium clostridioforme; B. coagulans = Bacillus coagulans.

trial. A total of 266 subjects with periodontitis were enrolled at seven treatment centers, with 227 subjects completing the study. Compliance with study medication was very high (>92\% in total); therefore, we confirm that by decreasing dosing frequencies, compliance is improved (compared to twice-daily dosing ${ }^{5}$ ). Furthermore, SDD-40 was tolerated very well by the subjects, with no AEs that were considered by the investigators to be probably related to study medication and no SAEs that were considered related to study treatments. There were

\section{DISCUSSION}

Previous investigations established the use of SDD (20 mg, twice a day) as an adjunct to non-surgical periodontal therapy. Improved treatment outcomes were reported in clinical studies of subjects with chronic periodontitis ${ }^{1,2,5}$ or severe, generalized chronic periodontitis ${ }^{3}$ and in at-risk groups, such as smokers ${ }^{24}$ and the institutionalized elderly. ${ }^{25}$ Reductions in GCF MMP levels were reported to coincide with improvements in clinical status following SDD treatment, ${ }^{4,11,12}$ and a benefit of SDD was reported when used as an adjunct to periodontal flap surgery. ${ }^{13}$ SDD is remarkably well tolerated, with no evidence of development of antibiotic resistance or any detrimental antimicrobial effects and no evidence of an adverse safety profile. ${ }^{1,5,6,8}$ Thus, SDD is a valid and effective adjunct in the treatment of periodontal disease and should be one of the options for patients to consider when deciding their treatment strategy in collaboration with their clinician. Although compliance rates were reported to be high $(>87 \%)$ in a clinical trial ${ }^{5}$ of the 20-mg formulation "IT of SDD taken twice daily, it is well known in the medical community that compliance and patient preference are increased if the frequency of daily dosing is decreased. ${ }^{17-19}$ Therefore, a once-daily 40 -mg modified-release formulation of SDD (SDD-40) as an adjunct for the treatment of periodontitis was developed to improve patient compliance while maintaining a consistent therapeutic benefit.

The once-daily 40-mg dose (SDD-40) was compared to placebo in this 9-month, randomized clinical no significant differences in the numbers or types of AEs between treatment groups, including those associated with the gastrointestinal tract or skin (particularly rash, photosensitivity reactions, and sunburn). Surveillance for these latter categories of possible AEs was particularly high, given the known pharmacology of doxycycline at antibiotic doses $\geq 100 \mathrm{mg}$.

As indicated in Table 1, the mean age of subjects recruited to the study was $\sim 48$ to 50 years; although the inclusion criteria allowed for subjects $\geq 18$ years to be recruited, the vast majority of those enrolled would be assigned a diagnosis of chronic periodontitis using the current classification system. ${ }^{26}$ Subjects were recruited to the study based on the extent and severity of periodontitis, as specified in the inclusion criteria.

Similar to previous studies of twice-daily $20-\mathrm{mg}$ formulations of SDD, ${ }^{1-3,5}$ improvements in mean CAL and PD were significantly greater in the adjunctive SDD-40 group than in the placebo group at every follow-up examination $(P<0.05)$. Consistent with observations that the magnitude of PD reductions and CAL gains following SRP is related to the pretreatment $\mathrm{PD},{ }^{21}$ in our study, the improvements in mean PD and CAL were greater in sites with pretreatment $P D \geq 7 \mathrm{~mm}$ compared to those with pretreatment PD of 4 to $6 \mathrm{~mm}$. In the deepest sites, at 9 months, mean CAL gains were $19 \%$ greater and mean PD reductions were $22 \%$ greater with adjunctive SDD-40 compared to adjunctive placebo, representing a tangible clinical benefit for subjects. It was reported that following SRP

II Periostat, CollaGenex Pharmaceuticals. 
alone in deep sites (PD $\geq 7 \mathrm{~mm}$ ), mean PD reductions $\sim 2.16 \mathrm{~mm}$ and attachment gains $\sim 1.19 \mathrm{~mm}$ can be expected, ${ }^{21,27}$ whereas in our study, in subjects treated with SRP and SDD-40, substantially greater clinical improvements were identified in mean PD reductions (2.74 mm at month 9) and mean CAL gains $(2.62 \mathrm{~mm}$ at month 9). In moderate sites (PD 4 to $6 \mathrm{~mm}$ ), mean PD reductions $\sim 1.29 \mathrm{~mm}$ and mean CAL gains $\sim 0.55$ $\mathrm{mm}$ can be expected following SRP alone, ${ }^{21,27}$ whereas we identified mean CAL gains of $1.66 \mathrm{~mm}$ and mean PD reductions of $1.63 \mathrm{~mm}$ in the subjects treated with SRP and adjunctive SDD-40.

Compelling evidence to support the clinical benefit of using SDD-40 as an adjunct to SRP is presented in Table 3. Threshold analyses were undertaken to determine the proportion of sites in each group that demonstrated PD reductions and CAL gains $\geq 2$ and $\geq 3 \mathrm{~mm}$. These thresholds were chosen because they represent a tangible clinical benefit that can be identified easily by a clinician with a periodontal probe. Although changes in full-mouth mean PD and CAL measurements are useful summary statistics for research purposes, as clinicians, we never quantify a subject's treatment response in this way. Instead, we assess clinically meaningful changes in periodontal status, such as probing reductions above a certain threshold, e.g., $2 \mathrm{~mm}$. Therefore, the threshold analyses in this study were undertaken to assess the clinical significance of any benefits of SDD-40. Table 3 provides abundantly clear evidence of the huge clinical benefit of SDD-40 in this population. The benefit of SDD-40 is particularly apparent when considering deep sites that had PD $\geq 6 \mathrm{~mm}$ at the start of the study, with $\sim 72 \%$ to $76 \%$ of sites achieving PD reductions and CAL gains $\geq 2 \mathrm{~mm}$ in the SDD-40 group compared to $56 \%$ to $58 \%$ of sites achieving these improvements in the placebo group. When considering an even more stringent threshold of improvement of $\geq 3 \mathrm{~mm}, \sim 48 \%$ to $52 \%$ of sites in the SDD-40 group achieved PD reductions and CAL gains $\geq 3 \mathrm{~mm}$ compared to $32 \%$ of sites in the placebo group. These impressive data underscore the clinical benefit of adjunctive treatment with SDD-40.

In this study, significantly greater reductions in BOP were recorded in the SDD-40 group than in the placebo group at all time points in the moderate sites and at months 3 and 9 in the deep sites. Reductions in BOP are consistent with improvements in periodontal health; the absence of BOP is a good measure of periodontal stability, indicating resolution of inflammation, and is a predictor for the maintenance of periodontal health. ${ }^{28}$ In this study, we interpreted the significant reductions in BOP in the SDD-40 group as indicative of enhanced clinical improvements (manifested by greater PD reductions and CAL gains). Previous studies ${ }^{1,5}$ of the 20 -mg formulation of SDD did not tend to identify significant differences between treatment groups in BOP reductions, although this is likely due to the different methodology used. Those studies recorded BOP in a dichotomous fashion, which may have lacked the discriminatory ability to identify differences between groups compared to the use of a graduated bleeding index as in the present study. ${ }^{20}$

Consistent with previous studies, ${ }^{1,5}$ the number of periodontal sites that demonstrated ongoing periodontal breakdown (defined by loss of attachment $\geq 2 \mathrm{~mm}$ from baseline) was recorded, and appropriate treatment was implemented following consultation between the subject and the investigator. A small proportion of sites demonstrated progressing disease over the 9 months of the study (Table 5), and there was a consistent benefit of SDD-40 in that fewer sites with disease progression were identified. However, because of the small number of sites, this failed to achieve statistical significance.

From a microbiologic perspective, an objective of this study was to determine whether SDD-40 exerted any detectable effect on the subgingival flora associated with periodontally diseased sites. Pharmacokinetic studies demonstrated that once-daily SDD-40 yields steady-state serum levels $\sim 0.5$ to $0.6 \mu \mathrm{g} / \mathrm{ml}$ (CollaGenex Pharmaceuticals, Newtown, Pennsylvania; unpublished data), similar to the steady-state serum levels of twice-daily $20-\mathrm{mg}$ formulations of SDD that were reported previously. ${ }^{29}$ This level is considerably less than the MIC, determined in vitro using planktonic-grown cultures, for the vast majority of bacterial species isolated from the subgingival flora. Antibiotic levels necessary to inhibit these same bacterial species grown in climax biofilms are 100- to 500 -fold greater. ${ }^{30}$

However, the argument exists that sub-MIC levels of an antibiotic may result in an increase in resistance or the acquisition of resistance. To rule out this possibility, a comprehensive microbial examination of the subgingival flora was conducted immediately before the first dosage and after 9 months of dosage with SDD-40 or the placebo control. The effect of treatment was determined by evaluating: 1) the increase in resistance to doxycycline as an increase in the MIC required to inhibit the bacteria and in the proportion of the total bacteria recovered with resistance to the drug; 2) increases or decreases in periodontal and/or opportunistic pathogens; and 3) the development of cross- and/or multiantibiotic resistance. An imbalance existed at baseline between the two treatment groups so that statistical testing was not possible between groups at either sampling time point. However, when the data were analyzed for changes between baseline and month 9 , no statistical or microbiologically significant changes were detected in any 
of the parameters enumerated. The reason for the imbalance between treatment groups at baseline is not immediately clear. Variations between groups of subjects may arise from biologic variation arising purely by chance, sample methodology, or seasonal changes. However, there is no evidence in this study that any discernible factors could account for the differences between the SDD-40 and placebo groups at baseline, which we attribute purely to chance.

The results of the microbiologic analyses are in agreement with previously published findings ${ }^{6-8,23,31}$ concerning $20-\mathrm{mg}$, twice-daily formulations of SDD. They demonstrate that long-term use of SDD-40 does not result in a change in the microbial flora, an increase in the proportion of the flora resistance to 4 $\mu \mathrm{g} / \mathrm{ml}$ doxycycline, an increase or overgrowth by periodontal or opportunistic pathogens, an increase in resistance to doxycycline by any individual bacterial species, the acquisition of doxycycline resistance by bacteria previously susceptible, or the emergence of cross-resistance or multiantibiotic resistance.

\section{CONCLUSIONS}

This study demonstrated that adjunctive SDD-40 taken for 9 months resulted in significantly greater mean CAL gains and mean PD reductions compared to those achieved by SRP alone during this time period. More importantly, the clinical significance of adjunctive SDD-40 was confirmed by threshold analyses of the proportions of sites in each group achieving clinically relevant PD reductions and CAL gains $\geq 2$ and $\geq 3 \mathrm{~mm}$. These data are consistent with previous studies $^{1,5}$ that showed a clinical superiority when twice-daily 20-mg formulations of SDD were used as an adjunct to SRP. Also, similar to studies ${ }^{1,5}$ of 20-mg doses of SDD, SDD-40 was well tolerated, and the number, type, and severity of AEs encountered were similar in the SDD-40 and placebo groups. The data suggest that compliance was improved with the once-daily formulation of SDD-40 compared to previous studies of $20-\mathrm{mg}$ doses, although this needs to be confirmed in future studies. Again, similar to studies $^{6-8}$ of 20-mg doses of SDD, there was no evidence of any long-term treatment effect of SDD-40 on the microbial flora, including any development of antibiotic resistance. The data from this study confirm that SDD-40 is a safe and effective therapy and support its use as an adjunct to SRP in the management of subjects with periodontitis.

\section{ACKNOWLEDGMENTS}

This study was supported by a grant from CollaGenex Pharmaceuticals, Newtown, Pennsylvania. Dr. Bradshaw has been a paid consultant for CollaGenex, and Dr. Walker currently serves as a consultant for CollaGenex. Dr. Bradshaw is currently managing director of Global Consulting Partners - Medical Biometrics, Princeton, New Jersey and previously was global vice president of biometrics, data management, and clinical information technology at Covance, Princeton, New Jersey. Drs. Preshaw, Novak, Mellonig, Magnusson, Polson, Giannobile, Rowland, Thomas, Dawson, and Sharkey report no conflicts of interest related to this study.

\section{REFERENCES}

1. Caton JG, Ciancio SG, Blieden TM, et al. Treatment with subantimicrobial dose doxycycline improves the efficacy of scaling and root planing in patients with adult periodontitis. J Periodontol 2000;71:521-532.

2. Caton JG, Ciancio SG, Blieden TM, et al. Subantimicrobial dose doxycycline as an adjunct to scaling and root planing: Post-treatment effects. J Clin Periodontol 2001;28:782-789.

3. Novak MJ, Johns LP, Miller RC, Bradshaw MH. Adjunctive benefits of subantimicrobial dose doxycycline in the management of severe, generalized, chronic periodontitis. J Periodontol 2002;73:762-769.

4. Emingil G, Atilla G, Sorsa T, Luoto H, Kirilmaz L, Baylas H. The effect of adjunctive low-dose doxycycline therapy on clinical parameters and gingival crevicular fluid matrix metalloproteinase- 8 levels in chronic periodontitis. J Periodontol 2004;75:106-115.

5. Preshaw PM, Hefti AF, Novak MJ, et al. Subantimicrobial dose doxycycline enhances the efficacy of scaling and root planing in chronic periodontitis: A multicenter trial. J Periodontol 2004;75:1068-1076.

6. Walker C, Thomas J, Nango S, Lennon J, Wetzel J, Powala C. Long-term treatment with subantimicrobial dose doxycycline exerts no antibacterial effect on the subgingival microflora associated with adult periodontitis. J Periodontol 2000;71:1465-1471.

7. Skidmore R, Kovach R, Walker C, et al. Effects of subantimicrobial-dose doxycycline in the treatment of moderate acne. Arch Dermatol 2003;139:459-464.

8. Walker C, Preshaw PM, Novak J, Hefti AF, Bradshaw $M$, Powala C. Long-term treatment with sub-antimicrobial dose doxycycline has no antibacterial effect on intestinal flora. J Clin Periodontol 2005;32:1163-1169.

9. Golub LM, Sorsa T, Lee HM, et al. Doxycycline inhibits neutrophil (PMN)-type matrix metalloproteinases in human adult periodontitis gingiva. J Clin Periodontol 1995;22:100-109.

10. Romanelli R, Mancini S, Laschinger C, Overall CM, Sodek J, McCulloch CA. Activation of neutrophil collagenase in periodontitis. Infect Immun 1999;67:2319. 2326.

11. Choi DH, Moon IS, Choi BK, et al. Effects of subantimicrobial dose doxycycline therapy on crevicular fluid MMP-8, and gingival tissue MMP-9, TIMP-1 and IL-6 levels in chronic periodontitis. $J$ Periodontal Res 2004;39:20-26.

12. Golub LM, Lee HM, Greenwald RA, et al. A matrix metalloproteinase inhibitor reduces bone-type collagen degradation fragments and specific collagenases in gingival crevicular fluid during adult periodontitis. Inflamm Res 1997;46:310-319.

13. Gapski R, Barr JL, Sarment DP, Layher MG, Socransky SS, Giannobile WV. Effect of systemic matrix metalloproteinase inhibition on periodontal wound repair: 
A proof of concept trial. $J$ Periodontol 2004;75: 441-452.

14. Brown DL, Desai KK, Vakili BA, Nouneh C, Lee HM, Golub LM. Clinical and biochemical results of the metalloproteinase inhibition with subantimicrobial doses of doxycycline to prevent acute coronary syndromes (MIDAS) pilot trial. Arterioscler Thromb Vasc Biol 2004;24:733-738.

15. O'Dell JR, Elliott JR, Mallek JA, et al. Treatment of early seropositive rheumatoid arthritis: Doxycycline plus methotrexate versus methotrexate alone. Arthritis Rheum 2006;54:621-627.

16. Bikowski JB. Subantimicrobial dose doxycycline for acne and rosacea. Skinmed 2003;2:234-245.

17. Jordan TJ, Reichman LB. Once-daily versus twicedaily dosing of theophylline. A decision analysis approach to evaluating theophylline blood levels and compliance. Am Reu Respir Dis 1989;140:1573-1577.

18. Andrejak M, Genes N, Vaur L, Poncelet P, Clerson P, Carre A. Electronic pill-boxes in the evaluation of antihypertensive treatment compliance: Comparison of once daily versus twice daily regimen. Am J Hypertens 2000;13:184-190.

19. Simon JA, Lewiecki EM, Smith ME, Petruschke RA, Wang L, Palmisano JJ. Patient preference for onceweekly alendronate $70 \mathrm{mg}$ versus once-daily alendronate $10 \mathrm{mg}$ : A multicenter, randomized, open-label, crossover study. Clin Ther 2002;24:1871-1886.

20. Polson AM, Southard GL, Dunn RL, Polson AP, Billen JR, Laster LL. Initial study of guided tissue regeneration in Class II furcation defects after use of a biodegradable barrier. Int $J$ Periodontics Restorative Dent 1995; 15:42-55.

21. Cobb CM. Clinical significance of non-surgical periodontal therapy: An evidence-based perspective of scaling and root planing. J Clin Periodontol 2002;29 (Suppl. 2):6-16.

22. Walker C, Sedlacek MJ. An in vitro biofilm model of subgingival plaque. Oral Microbiol Immun 2007;22: 152-161.
23. Thomas J, Walker C, Bradshaw M. Long-term use of subantimicrobial dose doxycycline does not lead to changes in antimicrobial susceptibility. J Periodontol 2000;71:1472-1483.

24. Preshaw PM, Hefti AF, Bradshaw MH. Adjunctive subantimicrobial dose doxycycline in smokers and non-smokers with chronic periodontitis. J Clin Periodontol 2005;32:610-616.

25. Mohammad AR, Preshaw PM, Bradshaw MH, Hefti AF, Powala CV, Romanowicz M. Adjunctive subantimicrobial dose doxycycline in the management of institutionalised geriatric patients with chronic periodontitis. Gerodontology 2005;22:37-43.

26. Armitage GC. Development of a classification system for periodontal diseases and conditions. Ann Periodontol 1999;4:1-6.

27. Cobb CM. Non-surgical pocket therapy: Mechanical. Ann Periodontol 1996;1:443-490.

28. Lang NP, Nyman S, Adler R, Joss A. Absence of bleeding on probing - A predictor for periodontal health. $J$ Clin Periodontol 1990;17:714-721.

29. Caton JG. Evaluation of Periostat for patient management. Compend Contin Educ Dent 1999;20:451-456.

30. Walker CB, Karpinia K, Baehni P. Chemotherapeutics: Antibiotics and other antimicrobials. Periodontol 2000 2004;36:146-165.

31. Thomas JG, Metheny RJ, Karakiozis JM, Wetzel JM, Crout RJ. Long-term sub-antimicrobial doxycycline (Periostat) as adjunctive management in adult periodontitis: Effects on subgingival bacterial population dynamics. Adv Dent Res 1998;12:32-39.

Correspondence: Dr. Philip M. Preshaw, Department of Periodontology, School of Dental Sciences, Newcastle University, Framlington Place, Newcastle upon Tyne, NE2 4BW, U.K. Fax: 44-0191-232-5144; e-mail: p.m.preshaw@ ncl.ac.uk.

Submitted July 3, 2007; accepted for publication September 5, 2007. 